\title{
Interference-free SDMA for FBMC-OQAM
}

\author{
François Horlin ${ }^{1 *}$, Jessica Fickers ${ }^{1}$, Thibault Deleu ${ }^{1}$ and Jérome Louveaux ${ }^{2}$
}

\begin{abstract}
Filter-bank multi-carrier (FBMC) modulations have recently been considered for the emerging wireless communication systems as a means to improve the utilization of the physical resources and the robustness to channel time variations. FBMC divides the overall frequency channel in a set of subchannels of bandwidth proportionally decreasing with the number of subchannels. If the number of subchannels is high enough, the bandwidth of each subchannel is small enough to assume that it is approximately flat. On the other hand, space-division multiple access (SDMA) is a recognized technique to support multiple access in the downlink of a multi-user system. The user signals are precoded at the base station equipped with multiple antennas to separate the users in the spatial domain. The application of SDMA to FBMC is unfortunately difficult when the channel is too frequency selective (or when the number of subchannels to too small) to assume flat subchannels. In that case, the system suffers from inter-symbol and inter-subchannel interference, besides the multi-user interference inherent to SDMA. State-of-the art solutions simply neglect the inter-symbol/subchannel interference. This article proposes a new SDMA precoder for FBMC capable of mitigating the three sources of interference. It is constructed per subchannel in order to keep an acceptable complexity and has the structure of a filter applied on each subchannel and its neighbors at twice the symbol rate. Numerical results demonstrate that the precoder can get rid of all the interference present in the system and benefit therefore from the diversity and power gains achievable with multiple antenna systems.
\end{abstract}

Keywords: Wireless communications, Multi-user downlink, Filter-bank modulations, FBMC, OFDM-OQAM, Multiple antenna technologies, MIMO SDMA

\section{Introduction}

The emerging wireless communication systems are carefully designed to simultaneously optimize the delivered user capacity, the average spectral efficiency and the cell coverage. Nowadays most of the emerging communications systems rely on the orthogonal frequencydivision multiplexing (OFDM) modulation technique [1]. By adding a redundant cyclic prefix to the symbol sequences, OFDM creates orthogonal and flat subchannels in the frequency domain that can be equalized at a low complexity by single coefficient inversion. The OFDM modulation has also been selected because it is easily combined with multi-input multi-output (MIMO) techniques exploiting the extra spatial dimension besides the time and frequency dimensions to increase the link reliability and capacity $[2,3]$. The MIMO techniques are applied

\footnotetext{
*Correspondence: francois.horlin@ulb.ac.be

1 OPERA Department, Université libre de Bruxelles, Av. Roosevelt 50,

1050 Brussels, Belgium

Full list of author information is available at the end of the article
}

on each subchannel independently in order to keep a low computational complexity.

Filter-bank multi-carrier (FBMC) modulations, and more specifically FBMC-offset quadrature amplitude modulation (OQAM), are seen as an interesting alternative to OFDM for future wireless communication systems $[4,5]$. The time/frequency resolution of the waveforms is increased and can be traded-off resulting in a better utilization of the physical resources and potentially in an improved robustness to time-variant channel characteristics and carrier frequency offsets. Like OFDM, FBMC-OQAM decomposes the communication channel in a set of lower-bandwidth subchannels that can therefore also be compensated at a low complexity with a single-tap equalizer. Contrary to OFDM, FBMC-OQAM does not require the addition of a cyclic prefix and the created subchannels are only approximately flat and orthogonal. When the channel frequency selectivity increases, the FBMC-OQAM system suffers from both inter-subchannel interference and inter-symbol interference on each subchannel, making it necessary to use

\section{照 Springer}

C 2013 Horlin et al: licensee Springer. This is an Open Access article distributed under the terms of the Creative Commons

Attribution License (http://creativecommons.org/licenses/by/2.0), which permits unrestricted use, distribution, and reproduction in any medium, provided the original work is properly cited. 
advanced equalizer structures [6]. Furthermore the combination of FBMC-OQAM with MIMO techniques results in an unmanageable interference term appearing between the antenna streams on adjacent subchannels, that makes the design of the system challenging. The design of MIMO FBMC-OQAM systems has triggered a lot of research recently.

The FBMC-OQAM system can first be combined with spatial division multiplexing (SDM) used to increase the link capacity. At the transmitter, different symbol streams are sent independently on the subchannels of the different antennas. Article [7] proposes to compensate for the inter-subchannel interference generated by the multiantenna streams at the receiver with an equalizer working per subchannel at a fractional symbol rate. While [7] focuses on linear and successive interference cancelation equalizers, article [8] extends the results to the adaptive decision feedback equalizer. To better cope with intersubchannel interference in the SDM system, a modified symbol mapping is also proposed in $[9,10]$ and combined with maximum-likelihood (ML) detection.

The FBMC-OQAM is secondly combined with the space-time block codes (and specifically with the Alamouti scheme) and with the space-time trellis codes to improve the communication reliability. Article [11] proposes to apply the Alamouti scheme designed initially for a flat fading channel to each subchannel independently at the transmitter and to handle the inter-subchannel interference generated by the multi-antenna streams at the receiver with an additional equalizer. Article [12] rather combines FBMC-OQAM with code-division multiple access (CDMA) to restore the complex orthogonality among the subchannels and be therefore able to apply and decode the Alamouti scheme independently per-subchannel. Finally [13] applies the space-time trellis codes to FBMC-OQAM and combines it with iterative decoding at the receiver.

In the article [14] and in the report of the European project Phydyas [15], it is proposed to perform transmit beamforming by multiplying each subchannel at the transmitter with a complex coefficient. An equalizer is further foreseen at the receiver to compensate for the inter-symbol and inter-subchannel interferences.

In systems where the base station is equipped with multiple antennas, it is also possible to provide multiple access in the spatial domain. This multi-user technique is referred to as space-division multiple access (SDMA). In line-of-sight environments, SDMA can be conceived as a form of beamsteering where different beams are aimed at different users, thereby separating their signals. In non-line-of-sight environments, wideband beamforming is required. Combined with OFDM, wideband SDMA reduces to narrowband beamforming per-subchannel $[16,17]$. Combined with FBMC-OQAM, wideband SDMA can also be implemented with a singletap beamformer per-subchannel by neglecting the intersymbol and inter-subchannel interferences. However, the level of remaining interference can significantly reduce the performance if an additional equalizer structure is not present.

In this article, we design a wideband SDMA linear precoder that separates the users spatially in the downlink of an FBMC-OQAM system. Compared with the existing literature, the main contribution of this article is to propose a structure that is able to suppress all sources of interference present in the FBMC-OQAM system: inter-symbol, inter-subchannel, and multi-user interference; even when the subchannels are frequency selective (highly selective channels, low number of subchannels). In order to do so, we present a structure where the precoder is a filter applied at twice the symbol rate, on each subchannel and its two adjacent subchannels. The computational complexity of the resulting precoder is linearly increasing with the number of subchannels and is thus still moderate. The SDMA precoder is built based on the knowledge of the channel impulse responses according to the zero-forcing criterion (inversion of the frequency selective channel).

The article is organized as follows. Section 2 introduces the model of the FBMC-OQAM system. The SDMA precoder is built in Section 3 based on a unified system model, detailing the structure of the precoder and showing how it can be computed in practice. Finally Section 4 demonstrates the efficiency of the scheme numerically. In the sequel, we use bold lowercase letters for the vectors and bold uppercase letters for the matrices. The operators $(.)^{T}$ and $(.)^{H}$ are used to denote the transpose and conjugate transpose of a vector or a matrix. Matrix $\mathbf{0}_{M, N}$ is the size- $M \times N$ matrix composed of zeros, matrix $\mathbf{I}_{N}$ is the size- $N$ identity matrix.

\section{System model}

\subsection{FBMC-OQAM system}

The article investigates the downlink of a SDMA system: a multi-antenna base station transmits independent signals to multiple users. The number of antennas $N_{T}$ at the base station is assumed equal to or larger than the number of users $N_{R}$. To generate the signal at the antenna $n_{T}$, the base station implements a synthesis filterbank composed of $M$ subchannels. Figure 1 details the model of the SDMA FBMC-OQAM system, focusing on antenna $n_{T}$ at the base station $\left(n_{T}=1, \ldots, N_{T}\right)$, on receiver $n_{R}\left(n_{R}=\right.$ $\left.1, \ldots, N_{R}\right)$, and on subchannel $k$ and its two adjacent subchannels $k-1$ and $k+1(k=-M / 2, \ldots, M / 2-1)$.

In the conventional FBMC-OQAM system, the sequence of QAM information symbols $s_{\left(n_{T}, k\right)}[l]$ transmitted on each subchannel $k$ at the symbol rate $R_{s}=1 / T_{s}$ ( $T_{s}$ is the QAM symbol duration) is separated into its real 


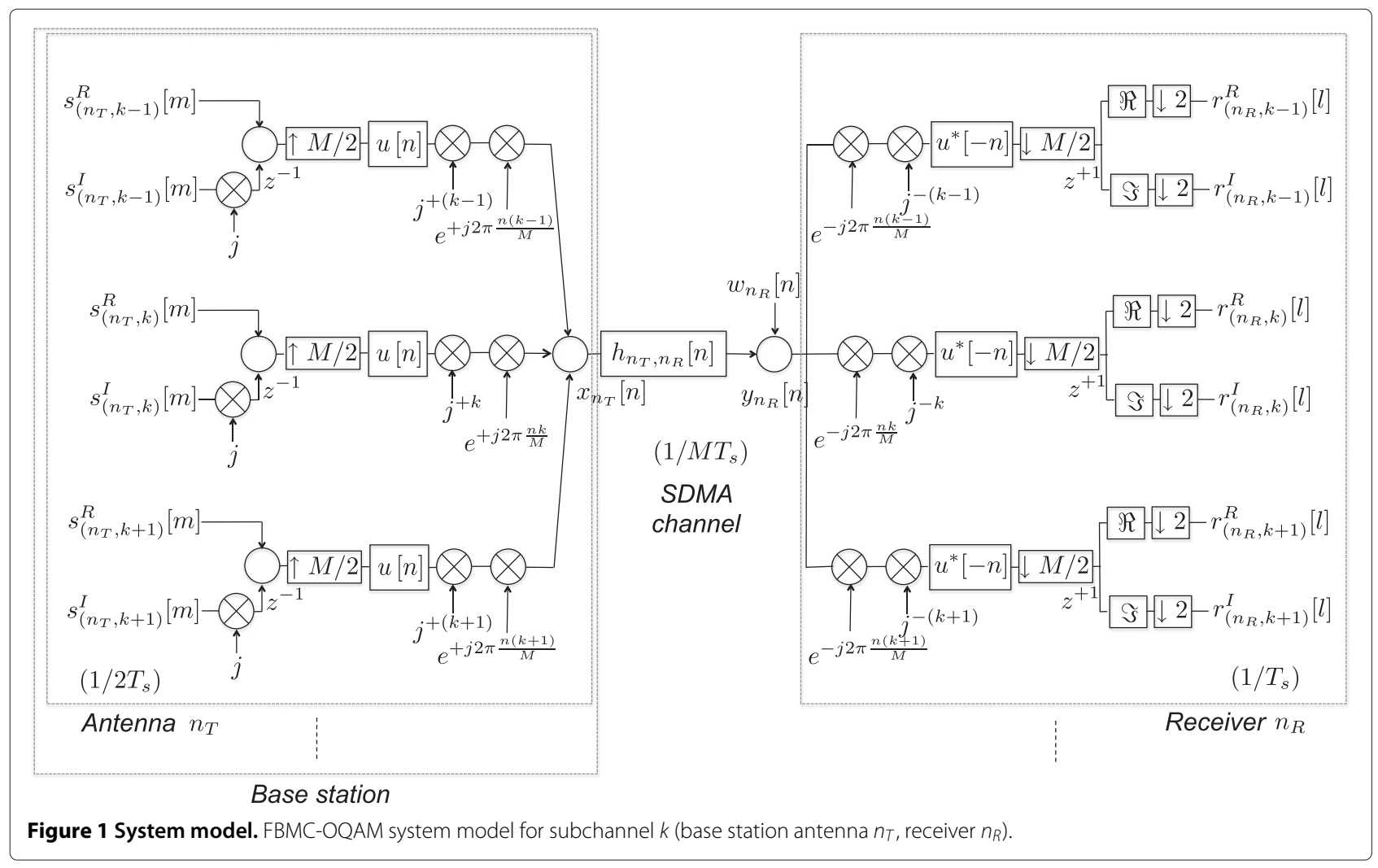

and imaginary parts, and upsampled by two to obtain the real sequences: $s_{\left(n_{T}, k\right)}^{R}[m]$ and $s_{\left(n_{T}, k\right)}^{I}[m]$ :

$$
\begin{aligned}
& s_{\left(n_{T}, k\right)}^{R}[m]:=\left\{\begin{array}{cl}
\Re\left[s_{\left(n_{T}, k\right)}[l]\right] & , m=2 l \\
0 & , \text { else }
\end{array}\right. \\
& s_{\left(n_{T}, k\right)}^{I}[m]:=\left\{\begin{array}{cl}
\Im\left[s_{\left(n_{T}, k\right)}[l]\right] & , m=2 l \\
0 & , \text { else } .
\end{array}\right.
\end{aligned}
$$

To implement the OQAM modulation, the sequence $s_{\left(n_{T}, k\right)}^{I}[m]$ is delayed by one sample (half a symbol), multiplied by $j$, and summed with the sequence $s_{\left(n_{T}, k\right)}^{R}[m]$. In this article, we design a SDMA precoder that generates the sequences $s_{\left(n_{T}, k\right)}^{R}[m]$ and $s_{\left(n_{T}, k\right)}^{I}[m]$ directly at its output. Because, the sequences $s_{\left(n_{T}, k\right)}^{R}[m]$ and $s_{\left(n_{T}, k\right)}^{I}[m]$ are defined at twice the symbol rate, the SDMA prefilter is built on the frequency band $\left[-1 / T_{s}, 1 / T_{s}\right]$.

After upsampling by $M / 2$ to reach the sampling rate $1 / M T_{s}$, the symbols are convolved with the pulse shaping filter $u[n]$. Without loss of generality, we assume that the pulse shaping filter $u[n]$ is a halfroot Nyquist filter of cut-off frequency equal to the symbol rate. In practice, the pulse shaping filter is often causal and of finite length. The model focuses on the subchannel $k$ and its two adjacent subchannels $k-1$ and $k+1$ as they are the only ones to impact the subchannel $k$. A factor $\pm j$ multiplies the signal on the odd subchannels. The $M$ subchannels are added together after signal shifting on the frequency grid. Two adjacent subchannels are spaced by a frequency shift equal to the input symbol rate.

The sequence transmitted at antenna $n_{T}$ is therefore given by:

$$
\begin{aligned}
x_{n_{T}}[n]= & \sum_{k=-M / 2}^{M / 2-1}(j)^{k} \exp \left(j 2 \pi \frac{n k}{M}\right) \\
& \times \sum_{m}\left(s_{\left(n_{T}, k\right)}^{R}[m]+j s_{\left(n_{T}, k\right)}^{I}[m-1]\right) u \\
& \times\left[m-n \frac{M}{2}\right] .
\end{aligned}
$$

Each user receives the sum of the signals transmitted from the $N_{T}$ base station antennas. The signal from antenna $n_{T}$ is received at the receiver $n_{R}$ after propagating through the frequency selective channel $h_{n_{T}, n_{R}}[n]$. Additive white Gaussian noise $w_{n_{R}}[n]$ of variance $\sigma_{w}^{2}$ corrupts the received sequence $y_{n_{R}}[n]$ at the receiver $n_{R}$. At each receiver, the analysis filterbank implements the counterpart of each operation performed at the transmitter. After frequency shifting to process each subchannel around the origin, the received signal is convolved with the filters 
matched to the pulse shaping filters. The half symbol delay applied on the imaginary branch is compensated so that both the real and imaginary signals are received synchronously when downsampling by $M$ to reach back the symbol rate $1 / T_{s}$. Only the real part on the real branch and the imaginary part on the imaginary branch are kept at the receiver.

In the case of a single-antenna/single-user system $\left(N_{T}=N_{R}=1\right)$, no interference is left at the output of the receiver when the channels are flat or slightly frequency selective thanks to the OQAM modulation and to the perfect reconstruction property of the pulse shaping filter. Therefore, a single slightly frequency selective channel can be compensated at a low complexity by simple channel coefficient inversion (like in OFDM). In the case of a multi-antenna system communicating over highly frequency selective channels, the system unfortunately suffers from three sources of interference: (i) inter-symbol interference on each subchannel; (ii) interference between the adjacent subchannels; (iii) inter-antenna interference. The goal of the SDMA precoder is to compensate for the multi-antenna frequency selective channel by prefiltering the information symbols so that the three sources of interference disappear.

\subsection{Equivalent system model}

The real output sequences $r_{\left(n_{R}, k\right)}^{R}[l]$ and $r_{\left(n_{R}, k\right)}^{I}[l]$ at the receiver $n_{R}$ on subchannel $k$ can be expressed as a function of the real input sequences $s_{\left(n_{T}, i\right)}^{R}[m]$ and $s_{\left(n_{T}, i\right)}^{I}[m]$ coming from all transmit antennas $\left(n_{T}=1, \ldots, N_{T}\right)$ on the subchannel $k$ and its adjacent subchannels $(i=k-1, k, k+$ $1)$. Since we are developing the precoder at twice the symbol rate, we need to define the polyphase components of the input sequences:

$$
\begin{aligned}
& s_{\left(n_{T}, i\right), \rho}^{R}[l]:=s_{\left(n_{T}, i\right)}^{R}[2 l+\rho] \\
& s_{\left(n_{T}, i\right), \rho}^{I}[l]:=s_{\left(n_{T}, i\right)}^{I}[2 l+\rho]
\end{aligned}
$$

for $\rho=0,1$ so that the overall model is eventually working at the symbol rate:

$$
\begin{aligned}
r_{\left(n_{R}, k\right)}^{R}[l]= & \sum_{n_{T}=1}^{N_{T}} \sum_{i=k-1}^{k+1} g_{\left(n_{T}, i\right),\left(n_{R}, k\right), 0}^{R, R}[l] \otimes s_{\left(n_{T}, i\right), 0}^{R}[l] \\
& +g_{\left(n_{T}, i\right),\left(n_{R}, k\right), 1}^{R, R}[l] \otimes s_{\left(n_{T}, i\right), 1}^{R}[l] \\
& +\sum_{n_{T}=1}^{N_{T}} \sum_{i=k-1}^{k+1} g_{\left(n_{T}, i\right),\left(n_{R}, k\right), 0}^{I, R}[l] \otimes s_{\left(n_{T}, i\right), 0}^{I}[l] \\
& +g_{\left(n_{T}, i\right),\left(n_{R}, k\right), 1}^{I, R}[l] \otimes s_{\left(n_{T}, i\right), 1}^{I}[l] \\
& +v_{\left(n_{R}, k\right)}^{R}[l]
\end{aligned}
$$

$$
\begin{aligned}
r_{\left(n_{R}, k\right)}^{I}[l]= & \sum_{n_{T}=1}^{N_{T}} \sum_{i=k-1}^{k+1} g_{\left(n_{T}, i\right),\left(n_{R}, k\right), 0}^{R, I}[l] \otimes s_{\left(n_{T}, i\right), 0}^{R}[l] \\
& +g_{\left(n_{T}, i\right),\left(n_{R}, k\right), 1}^{R, I}[l] \otimes s_{\left(n_{T}, i\right), 1}^{R}[l] \\
& +\sum_{n_{T}=1}^{N_{T}} \sum_{i=k-1}^{k+1} g_{\left(n_{T}, i\right),\left(n_{R}, k\right), 0}^{I, I}[l] \otimes s_{\left(n_{T}, i\right), 0}^{I}[l] \\
& +g_{\left(n_{T}, i\right),\left(n_{R}, k\right), 1}^{I, I}[l] \otimes s_{\left(n_{T}, i\right), 1}^{I}[l] \\
& +v_{\left(n_{R}, k\right)}^{I}[l]
\end{aligned}
$$

where $\otimes$ is the convolution operator. The parameter $\rho$ is relative to the two polyphase components. The composite channel impulse responses result from the convolution of the transmitter filter, channel response and receiver filter, downsampled by a factor $M$, as defined in:

$g_{\left(n_{T}, i\right),\left(n_{R}, k\right), \rho}^{R, R}[l]:=\Re\left\{p_{i}^{R}[n] \otimes h_{n_{T}, n_{R}}[n] \otimes q_{k}^{R}[n]\right\}_{\mid n=l M-\rho \frac{M}{2}}$

$$
g_{\left(n_{T}, i\right),\left(n_{R}, k\right), \rho}^{I, R}[l]:=\Re\left\{p_{i}^{I}[n] \otimes h_{n_{T}, n_{R}}[n] \otimes q_{k}^{R}[n]\right\}_{\mid n=l M-\rho \frac{M}{2}}
$$

$g_{\left(n_{T}, i\right),\left(n_{R}, k\right), \rho}^{R, I}[l]:=\Im\left\{p_{i}^{R}[n] \otimes h_{n_{T}, n_{R}}[n] \otimes q_{k}^{I}[n]\right\}_{\mid n=l M-\rho \frac{M}{2}}$

$$
g_{\left(n_{T}, i\right),\left(n_{R}, k\right), \rho}^{I, I}[l]:=\Im\left\{p_{i}^{I}[n] \otimes h_{n_{T}, n_{R}}[n] \otimes q_{k}^{I}[n]\right\}_{\mid n=l M-\rho \frac{M}{2}}
$$

in which the functions $p_{k}^{R}[n], p_{k}^{I}[n]$ and $q_{k}^{R}[n], q_{k}^{I}[n]$ are the synthesis and analysis filters. They include the pulse shaping filter, the frequency shifts and the potential $j$ factor:

$$
\begin{aligned}
& p_{i}^{R}[n]:=u[n] \cdot(j)^{i} \cdot \exp \left(j 2 \pi \frac{i n}{M}\right) \\
& p_{i}^{I}[n]:=u[n-M / 2] \cdot(j)^{i+1} \cdot(-1)^{i} \cdot \exp \left(j 2 \pi \frac{i n}{M}\right)
\end{aligned}
$$

and:

$$
\begin{aligned}
& q_{i}^{R}[n]:=u^{*}[-n] \cdot(j)^{-i} \cdot \exp \left(j 2 \pi \frac{i n}{M}\right) \\
& q_{i}^{I}[n]:=u^{*}[-n-M / 2] \cdot(j)^{-i} \cdot(-1)^{i} \cdot \exp \left(j 2 \pi \frac{i n}{M}\right) .
\end{aligned}
$$


The noise sequences $v_{\left(n_{R}, k\right)}^{R}[l]$ and $v_{\left(n_{R}, k\right)}^{I}[l]$ are obtained by filtering the input noise sequence $w_{n_{R}}[n]$ with the analysis filters, downsampling by $M$, and keeping the real part of the result on the real branch or the imaginary part of the result on the imaginary branch.

It is noteworthy to observe by inspecting Figure 1 that:

$$
\begin{aligned}
& g_{\left(n_{T}, i\right),\left(n_{R}, k\right), \rho}^{R, R}[l]=g_{\left(n_{T}, i\right),\left(n_{R}, k\right), \rho}^{I, I}[l] \\
& g_{\left(n_{T}, i\right),\left(n_{R}, k\right), \rho}^{I, R}[l]=-g_{\left(n_{T}, i\right),\left(n_{R}, k\right), \rho}^{R, I}[l-1] .
\end{aligned}
$$

Because $g_{\left(n_{T}, i\right),\left(n_{R}, k\right), \rho}^{I, R}[l] \neq-g_{\left(n_{T}, i\right),\left(n_{R}, k\right), \rho}^{R, I}[l]$, it is impossible to build a unified complex model equivalent to (6), (7). Therefore, we have to stick to the real convolution model (6), (7) to correctly represent the FBMC-OQAM system (and the precoder that will be derived in the next sections).

The equivalent system model is illustrated in the right part of Figure 2. In order to simplify the diagram, the real/imaginary parts of the input sequence polyphase components at all transmit antennas have been arranged in a sequence of length- $4 N_{T}$ vectors $(i=k-1, k, k+1)$ :

$$
\begin{aligned}
& \mathbf{s}_{i}[l]:=\left[\begin{array}{llll}
s_{(1, i), 0}^{R}[l] & s_{(1, i), 1}^{R}[l] & \cdots & s_{\left(N_{T}, i\right), 1}^{R}[l]
\end{array}\right. \\
& \left.s_{(1, i), 0}^{I}[n] \quad s_{(1, i), 1}^{I}[l] \quad \cdots \quad s_{\left(N_{T}, i\right), 1}^{I}[l]\right]^{T},
\end{aligned}
$$

the real/imaginary parts of the noise and received sequences at all receive antennas have been arranged in sequences of length- $2 N_{R}$ vectors:

$$
\mathbf{r}_{k}[l]:=\left[r_{(1, k)}^{R}[l] \cdots r_{\left(N_{R}, k\right)}^{R}[l] \mid r_{(1, k)}^{I}[l] \cdots r_{\left(N_{R}, k\right)}^{I}[l]\right]^{T}
$$

$$
\mathbf{v}_{k}[l]:=\left[v_{(1, k)}^{R}[l] \cdots v_{\left(N_{R}, k\right)}^{R}[l] \mid v_{1, k}^{I}[l] \cdots v_{\left(N_{R}, k\right)}^{I}[l]\right]^{T}
$$

and the real/imaginary parts of the channel impulse responses corresponding to all transmit/receive anten-

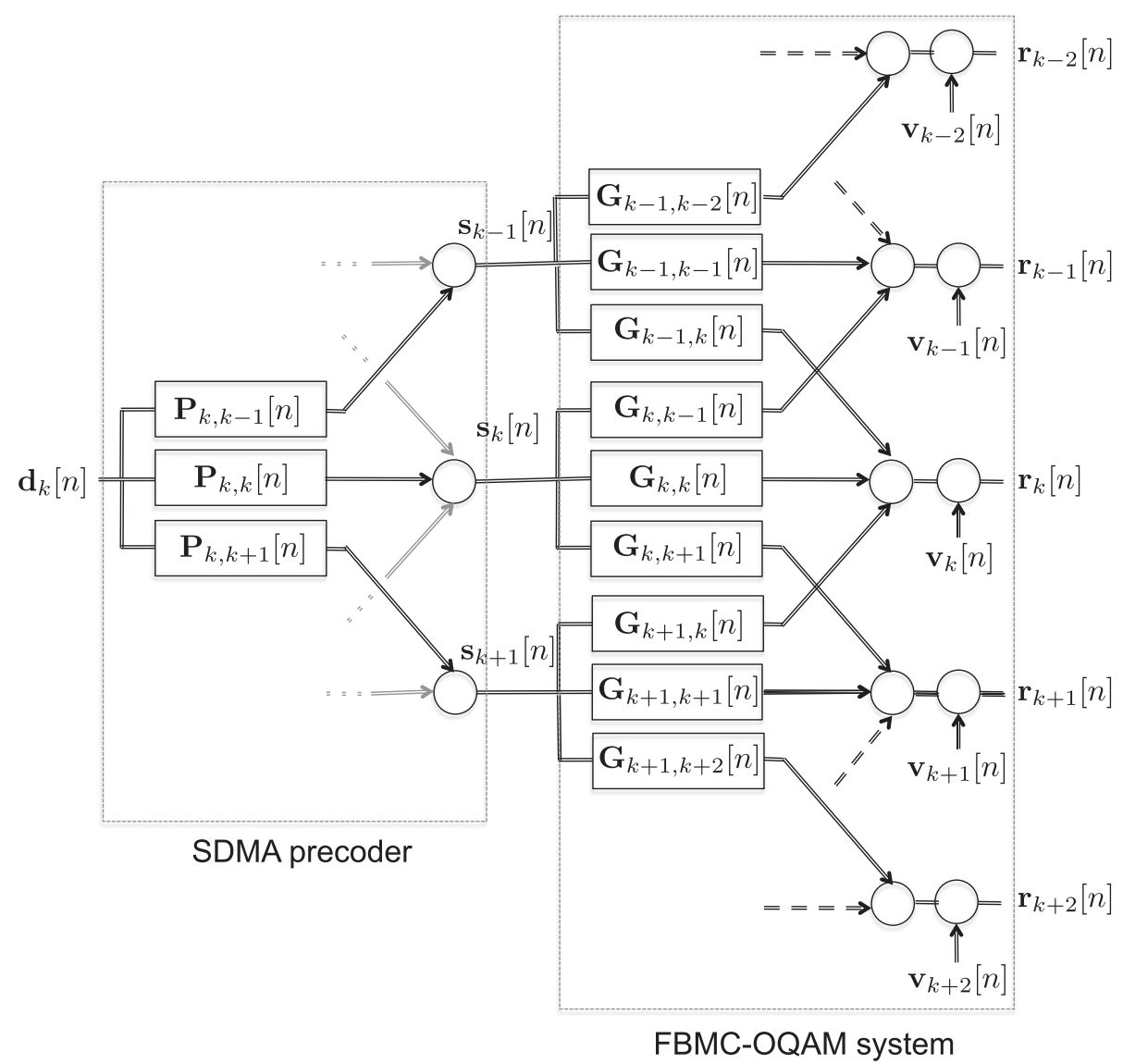

Figure 2 Equivalent system model. SDMA precoder for subchannel $k$ (left part); equivalent FBMC-OQAM system model (right part). 
nas are arranged in a sequence of size- $2 N_{R} \times 4 N_{T}$ matrices:

$$
\mathbf{G}_{i, k}[l]:=\left[\begin{array}{c|c}
\mathbf{G}_{i, k}^{R, R}[l] & \mathbf{G}_{i, k}^{I, R}[l] \\
\hline \mathbf{G}_{i, k}^{R, I}[l] & \mathbf{G}_{i, k}^{I, I}[l]
\end{array}\right] .
$$

with:

$$
\begin{aligned}
& \mathbf{G}_{i, k}^{X, Y}[l] \\
& :=\left[\begin{array}{cccc}
g_{(1, i),(1, k), 0}^{X, Y}[l] & g_{(1, i),(1, k), 1}^{X, Y}[l] & \cdots & g_{\left(N_{T}, i\right),(1, k), 1}^{X, Y}[l] \\
\vdots & \vdots & \ddots & \vdots \\
g_{(1, i),\left(N_{R}, k\right), 0}^{X, Y}[l] & g_{(1, i),\left(N_{R}, k\right), 1}^{X, Y}[l] & \cdots & g_{\left(N_{T}, i\right),\left(N_{R}, k\right), 1}^{X, Y}[l]
\end{array}\right] .
\end{aligned}
$$

for $X, Y=R, I$. Expressions (6), (7) are equivalently written:

$$
\mathbf{r}_{k}[l]=\sum_{i=k-1}^{k+1} \mathbf{G}_{i, k}[l] \otimes \mathbf{s}_{i}[l]+\mathbf{v}_{k}[n]
$$

where the definition of the convolution $\otimes$ of a vector sequence $\mathbf{x}[l]$ with a matrix sequence $\mathbf{G}[l]$ is traditionally defined as: $\mathbf{y}[l]:=\sum_{m=-\infty}^{\infty} \mathbf{G}[m] \cdot \mathbf{x}[m-l]$.

\section{SDMA precoder}

The SDMA precoder could be jointly designed for all subchannels taking their mutual impact into account. However this option would be of prohibitive computational complexity and it is in fact possible to build a simple zero-forcing precoder independently for each subchannel. It is based on the property that each subchannel only interferes with its two adjacent subchannels (it has no frequency overlap with the others). Then, each precoder for each subchannel is designed in such a way that the interference it causes on the other subchannels is canceled out, as well as the inter-antenna interference and the intersymbol interference inside its own subchannel. In order to have enough degrees of freedom, the precoder is built at twice the symbol rate. This way, the system is ensured to be free of interference.

This section derives the SDMA precoder for subchannel $k(k=-M / 2, \ldots, M / 2-1)$. It is performed in two steps: we first detail the structure of the SDMA precoder for subchannel $k$ based on the equivalent system model and, we secondly show how it can be computed in practice.

\subsection{Structure of the SDMA precoder}

The SDMA precoder for the subchannel $k$ is illustrated in the left part of Figure 2. It precodes the real input symbol vector sequence:

$$
\mathbf{d}_{k}[l]:=\left[d_{(1, k)}^{R}[l] \cdots d_{\left(N_{R}, k\right)}^{R}[l] \mid d_{(1, k)}^{I}[l] \cdots d_{\left(N_{R}, k\right)}^{I}[l]\right]^{T}
$$

with the real matrix filters $\mathbf{P}_{k, k-1}[l], \mathbf{P}_{k, k}[l]$ and $\mathbf{P}_{k, k+1}[l]$ of size $4 N_{T} \times 2 N_{R}$ to determine their contributions $\mathbf{s}_{k-1}[l], \mathbf{s}_{k}[l]$ and $\mathbf{s}_{k+1}[l]$ to the subchannels $k-1, k$ and $k+1$, respectively, of the FBMC-OQAM system.

The SDMA precoder is designed to fulfill the following conditions (zero-forcing criterion):

- The input symbols on subchannel $k$ do not interfere with the subchannels $k-2$ and $k+2$ :

$$
\begin{aligned}
& \mathbf{G}_{k-1, k-2}[l] \otimes \mathbf{P}_{k, k-1}[l]=\mathbf{0}_{2 N_{R}, 2 N_{R}} \\
& \mathbf{G}_{k+1, k+2}[l] \otimes \mathbf{P}_{k, k+1}[l]=\mathbf{0}_{2 N_{R}, 2 N_{R}} .
\end{aligned}
$$

- The input symbols on subchannel $k$ do not interfere with the subchannels $k-1$ and $k+1$ :

$$
\mathbf{G}_{k-1, k-1}[l] \otimes \mathbf{P}_{k, k-1}[l]+\mathbf{G}_{k, k-1}[l] \otimes \mathbf{P}_{k, k}[l]=\mathbf{0}_{2 N_{R}, 2 N_{R}}
$$

$$
\mathbf{G}_{k+1, k+1}[l] \otimes \mathbf{P}_{k, k+1}[l]+\mathbf{G}_{k, k+1}[l] \otimes \mathbf{P}_{k, k}[l]=\mathbf{0}_{2 N_{R}, 2 N_{R}} .
$$

- There is no inter-symbol/inter-antenna interference on subchannel $k$ :

$$
\begin{aligned}
\mathbf{G}_{k-1, k}[l] & \otimes \mathbf{P}_{k, k-1}[l]+\mathbf{G}_{k, k}[l] \otimes \mathbf{P}_{k, k}[l]+\mathbf{G}_{k+1, k}[l] \\
& \otimes \mathbf{P}_{k, k+1}[l]=\mathbf{I}_{2 N_{R}} \delta[l]
\end{aligned}
$$

where $\delta[l]$ is the Dirac pulse.

It should be noted that the usual condition required to design the SDMA precoder for a flat fading channel as proposed in [14,15] is $\mathbf{G}_{k, k} \otimes \mathbf{P}_{k, k}=\mathbf{I}_{2 N_{R}}$ that involves only the second term of (29) independent of the time index. The SDMA precoder for the FBMC-OQAM system has to fulfill the five conditions (25)-(29) together mostly because of the inter-subchannel interference inherent to the system.

\subsection{Expression of the SDMA precoder}

The overall system relative to subchannel $k$, including the SDMA precoder and the channel as illustrated in Figure 2, can be mathematically expressed in a unified matrix model:

$$
\overline{\mathbf{r}}_{k}[l]=\mathbf{G}_{k}[l] \otimes \mathbf{P}_{k}[l] \otimes \mathbf{d}_{k}[l]+\overline{\mathbf{v}}_{k}[l]
$$

where the received and noise sequences on the different subchannels have been stacked in length-10N $N_{R}$ received and noise vectors:

$$
\begin{aligned}
& \overline{\mathbf{r}}_{k}[l]:=\left[\mathbf{r}_{k-2}^{T}[l] \mathbf{r}_{k-1}^{T}[l] \mathbf{r}_{k}^{T}[l] \mathbf{r}_{k+1}^{T}[l] \mathbf{r}_{k+2}^{T}[l]\right]^{T} \\
& \overline{\mathbf{v}}_{k}[l]:=\left[\mathbf{v}_{k-2}^{T}[l] \mathbf{v}_{k-1}^{T}[l] \mathbf{v}_{k}^{T}[l] \mathbf{v}_{k+1}^{T}[l] \mathbf{v}_{k+2}^{T}[l]\right]^{T},
\end{aligned}
$$


and the size- $10 N_{R} \times 12 N_{T}$ channel matrix is:

$$
\mathbf{G}_{k}[l]:=\left[\begin{array}{ccc}
\mathbf{G}_{k-1, k-2}[l] & \mathbf{0}_{2 N_{R}, 4 N_{T}} & \mathbf{0}_{2 N_{R}, 4 N_{T}} \\
\mathbf{G}_{k-1, k-1}[l] & \mathbf{G}_{k, k-1}[l] & \mathbf{0}_{2 N_{R}, 4 N_{T}} \\
\mathbf{G}_{k-1, k}[l] & \mathbf{G}_{k, k}[l] & \mathbf{G}_{k+1, k}[l] \\
\mathbf{0}_{2 N_{R}, 4 N_{T}} & \mathbf{G}_{k, k+1}[l] & \mathbf{G}_{k+1, k+1}[l] \\
\mathbf{0}_{2 N_{R}, 4 N_{T}} & \mathbf{0}_{2 N_{R}, 4 N_{T}} & \mathbf{G}_{k+1, k+2}[l]
\end{array}\right],
$$

and the size- $12 N_{T} \times 2 N_{R}$ precoder matrix is:

$$
\mathbf{P}_{k}[l]:=\left[\begin{array}{c}
\mathbf{P}_{k, k-1}[l] \\
\mathbf{P}_{k, k}[l] \\
\mathbf{P}_{k, k+1}[l]
\end{array}\right] .
$$

Model (30) focuses on the contribution of the symbols transmitted on subchannel $k$ to the received vectors on subchannels $k-2$ to $k+2$, and therefore neglects the contribution from the other subchannels. Since the SDMA precoder is designed to prevent interference from subchannel $k$ on the subchannels $k \pm 1, k \pm 2$, model (30) is however sufficient.

A closed-form expression of the zero-forcing (ZF) precoder, of infinite length in the general case, is conveniently given in the $z$-domain. Let $\mathcal{G}_{k}[z]$ denotes the $z$-transformed channel matrix. The ZF precoder matrix is expressed as [18]:

$$
\mathcal{P}_{k}[z]=\tilde{\mathcal{G}}_{k}[z] \cdot\left(\mathcal{G}_{k}[z] \cdot \tilde{\mathcal{G}}_{k}[z]\right)^{-1} \cdot \Theta
$$

where $\tilde{\mathcal{G}}_{k}[z]=\mathcal{G}_{k}^{H}\left[z^{-1}\right]$ is the parahermitian of $\mathcal{G}_{k}[z]$. Matrix $\Theta$ is composed of the $2 N_{R}$ central columns of the identity matrix $\mathbf{I}_{10 N_{R}}$ so that the part of the precoder relative to the information symbols on the subchannel $k$ is selected. Among all precoders satisfying the zero forcing criterion (25)-(29), the precoder (35) taking the form of the channel matrix pseudo-inverse maximizes the sum capacity if a global power constraint is assumed at the transmitter [19]. We normalize the precoder when its performance is assessed to satisfy the power constraint. The zero-forcing precoder applies a filter matched to the channel impulse responses preceded by the inversion of the overall channel responses to mitigate the interference. The matched filter can be seen as a wideband beamformer and the ZF precoder may hence bring the corresponding power and diversity gains (when there is only one receiver, it is equivalent to the maximum-ratio combining scheme that is well-known to benefit from the two types of gain).

Different methods exist to practically compute the ZF filter for a MIMO channel [18]. In this article, we have followed the time-domain method first described in [20] that relies on the construction of a matrix model of the convo- lution MIMO channel. It is detailed in the Appendix 1 for the particular case considered in this article. The method always produces a finite impulse response solution of accuracy increasing with the matrix model dimension (the parameter $N$ in the Appendix 1). The computation of the SDMA precoder requires the inversion of the channel correlation matrix which is always well-conditioned when the number of antennas at the transmitter $N_{T}$ is larger than or equal to the number of users $N_{R}$. It is also interesting to note that the SDMA precoder can be developed at the symbol rate (instead of at twice the symbol rate) when $N_{T} \geq 2 N_{R}$. However the number of users has to be much smaller than the number of antennas at the base station in that case which drastically reduces the system capacity.

\section{Performance and implementation complexity analysis}

The performance and the implementation complexity of the proposed SDMA precoder is evaluated numerically. The FBMC system is composed of a varying number of subchannels $(M=32,64,128)$, each modulated with offset quaternary phase shift keying (OQPSK) symbols shaped with a halfroot Nyquist filter. A 1 rolloff factor is considered and there is hence significant spectral overlap between the adjacent subchannels. The length of the halfroot Nyquist filters is fixed to 8 symbols. The sample rate at the output of the subchannels is equal to 64 Msamples/s, so that the symbol rate at the input of each subchannel is equal to 2, 1 , and $0.5 \mathrm{Msymbols} / \mathrm{s}$ for $M=$ 32,64 , and 128, respectively. The base station is equipped with 4 antennas and the number of receivers is varying from 1 to 4 . A frequency selective channel of exponentially decreasing power delay profile is assumed. The channel delay spread is equal to $0.0525 \mu$ s (3.36 samples). The results are averaged over 1000 channel realizations that are normalized in power on the average.

\subsection{Implementation complexity}

The computational complexity required to implement the FBMC-OQAM system with SDMA is assessed and compared to the one required to implement the SDMAOFDM system [16,17]. A distinction must be made between the operations required to compute the precoder and those required to precode the sequence of symbols.

Regarding the complexity required to compute the precoder:

- Since the precoder computation involves mainly the inversion of the size $12 N_{R} N$ real channel correlation matrix for each subchannel (see the Appendix 1 for the details) and is therefore $\mathcal{O}\left(N_{R}^{3} N^{3}\right)$, the parameter $N$ is a direct measure of the computational complexity required for the computation of the precoder. 
- In comparison, the SDMA-OFDM system requires the inversion of a size $N_{R}$ complex channel correlation matrix for each carrier and is therefore $\mathcal{O}\left(N_{R}^{3}\right)$.

Regarding the precoding complexity:

- The SDMA precoder on each subchannel can be seen as a size $12 N_{T} \times 2 N_{R}$ matrix filter of length equal to $N+L-1$, where $L$ is the fixed length of the channel impulse responses (see the Appendix 1 for the details). Therefore, $24 N_{T} N_{R}(N+L-1)$ multiply-andaccumulate (MAC) real operations are performed for each set of $N_{R}$ symbols on each subchannel.

- In comparison, the SDMA-OFDM system requires the multiplication with a size $N_{T} \times N_{R}$ matrix on each subchannel so that $N_{T} N_{R}$ MAC complex operations are performed for each set of $N_{R}$ symbols on each subchannel.

Therefore a special care needs to be taken in order to select the dimension of the matrix model used to compute the precoder (the parameter $N$ ). On the one hand, $N$ should be as large as possible to ensure the accuracy of the precoder computation. On the other hand, $N$ should be as small as possible to limit the computational complexity.

Figure 3 illustrates the mean square error (MSE) as a function of the parameter $N$ for a varying number of subchannels. The MSE is computed by assessing the error remaining between the overall channel impulse response (precoder and channel impulse response) and the dirac pulse $^{\mathrm{a}}$. The number of receivers has been fixed to 3 . Figure 3 first confirms that the accuracy of the precoder computation improves when $N$ is increased as the remaining MSE becomes negligible. The minimum value $N$ necessary to achieve the desired MSE can therefore be inferred from the figure. By comparing the solid curves in Figure 3, it is secondly observed that the MSE is only slightly reduced when the number of subchannels increases as each subchannel becomes approximately frequency flat (no improvement is observed for $M$ larger than 64). Therefore the computational complexity is approximately independent of the number of subchannels. The dashed curve in Figure 3 illustrates finally the MSE loss obtained when the roll-off (ro) factor of the halfroot Nyquist filters is equal to 0.5 (instead of 1 ). In that case, the dimension of the matrix model necessary to compute the SDMA precoder (and therefore the computational complexity) must significantly be increased to ensure the same accuracy. This is explained by the fact that the filters are less localized in the time domain.

Even if the complexity of the proposed SDMA FBMCOQAM system is much larger than the complexity of the corresponding SDMA-OFDM system, it increases linearly with the number of subchannels and is therefore acceptable compared to the complexity that would be necessary when the precoder is jointly designed on all subchannels. In the following performance results, the SDMA precoder is computed by building a dimension 20 time-domain matrix model to ensure the accuracy of the precoder

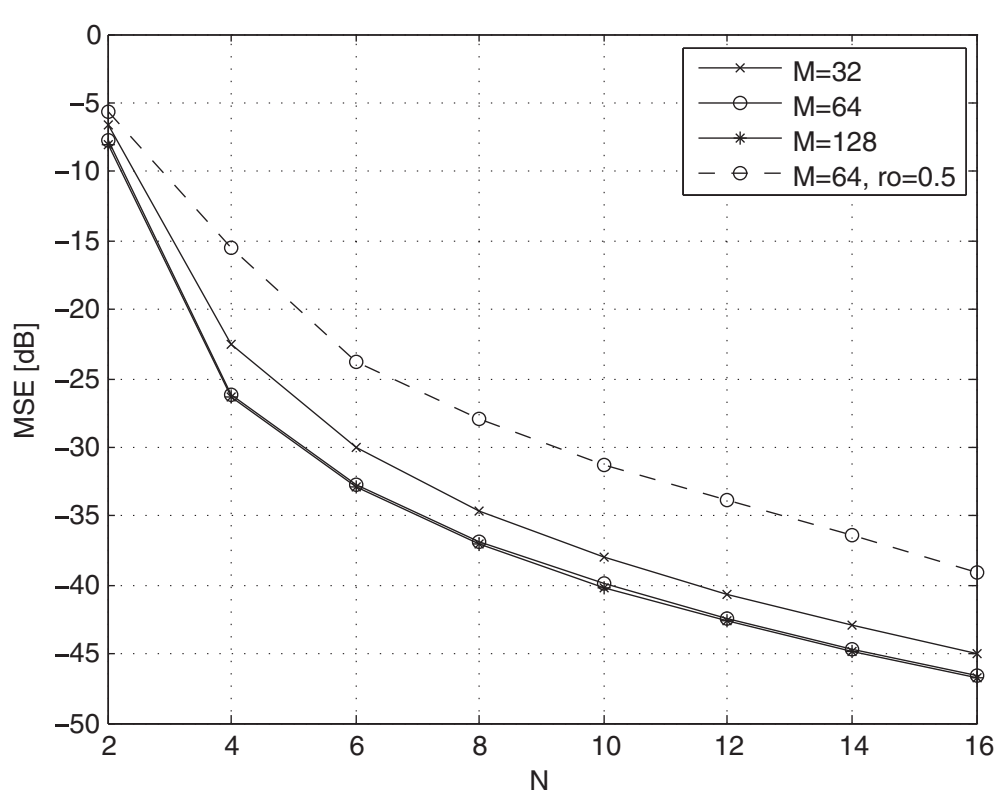

Figure 3 Precoder computation accuracy. Mean square error (MSE) as a function of the dimension $N$ of the matrix model used to compute the precoder for a varying number of subchannels. Solid lines: pulse shaping filter of roll-off 1 for $M$ varying; dashed line: pulse shaping filter of roll-off 0.5 for $M=64$. Parameters: $N_{T}=4, N_{R}=3$. 
computation. The length of the resulting precoder filters is approximately equal to 30 .

Another important system design parameter is the peak-to-average-power ratio (PAPR) that measures the implementation feasibility of the power amplifiers at the transmitter. Figure 4 illustrates the cumulative distribution of the PAPR for the FBMC-OQAM system with and without SDMA precoding. The comparison is first made by considering only 1 transmit antenna and 1 user, and then by extending the system to 4 transmit antennas and 3 users. The PAPR is assessed by comparing the peak power to the average power when bursts of 10 symbols are transmitted. It is observed in both cases that the SDMA precoder tends to decrease slightly the PAPR by introducing correlation between the signals on the adjacent channels.

\subsection{Performance analysis}

Figure 5 compares the bit error rate (BER) performance of the SDMA FBMC-OQAM system proposed in this article (solid curve) to the one of the SDMA-OFDM system investigated in $[16,17]$ (dashed curves). The BER performance is assessed as a function of the signal-to-noise power ratio (SNR) defined as the average transmitted power per antenna divided by the noise power in the system bandwidth (in practice, we normalize the precoder to fix the average transmitted power per antenna and vary the noise power). The number of receivers has been fixed to 3. The trade-off existing between the BER and the spectral efficiency of the OFDM system for a varying cyclic prefix $(\mathrm{CP})$ is investigated. When the $C P$ is null, the spectral efficiency of the OFDM system is maximum but there is significant inter-carrier interference (ICI), explaining why the curves tend asymptotically to a high BER value. When the CP is large (16 samples), there is no ICI anymore and the BER performance is similar to the one of the FBMC-OQAM system, but the spectral efficiency is reduced by $1 / 5$ compared to the FBMCOQAM system. A small power loss (1/5 of the power) is also observed compared to the FBMC-OQAM system because of the CP removal at the receiver, leading to a shift of the BER curve to the right. It is noteworthy that the ICI significantly limits the BER performance even when the $C P$ is slightly larger than the channel delay spread (4 samples). Compared to the SDMA-OFDM system, the proposed SDMA FBMC-OQAM system is capable of delivering the maximum spectral efficiency without any BER penalty.

Figure 6 illustrates the BER for a varying number of subchannels. The number of receivers is also fixed to 3 . The figure compares the performance of the proposed SDMA precoder (solid curves) to the one of the singletap SDMA precoder designed for the OFDM system, but used for the FBMC-OQAM system (dashed curves). Because the channel cannot be assumed to be flat on each subchannel, the single-tap SDMA precoder significantly suffers from the inter-symbol, inter-subchannel and inter-

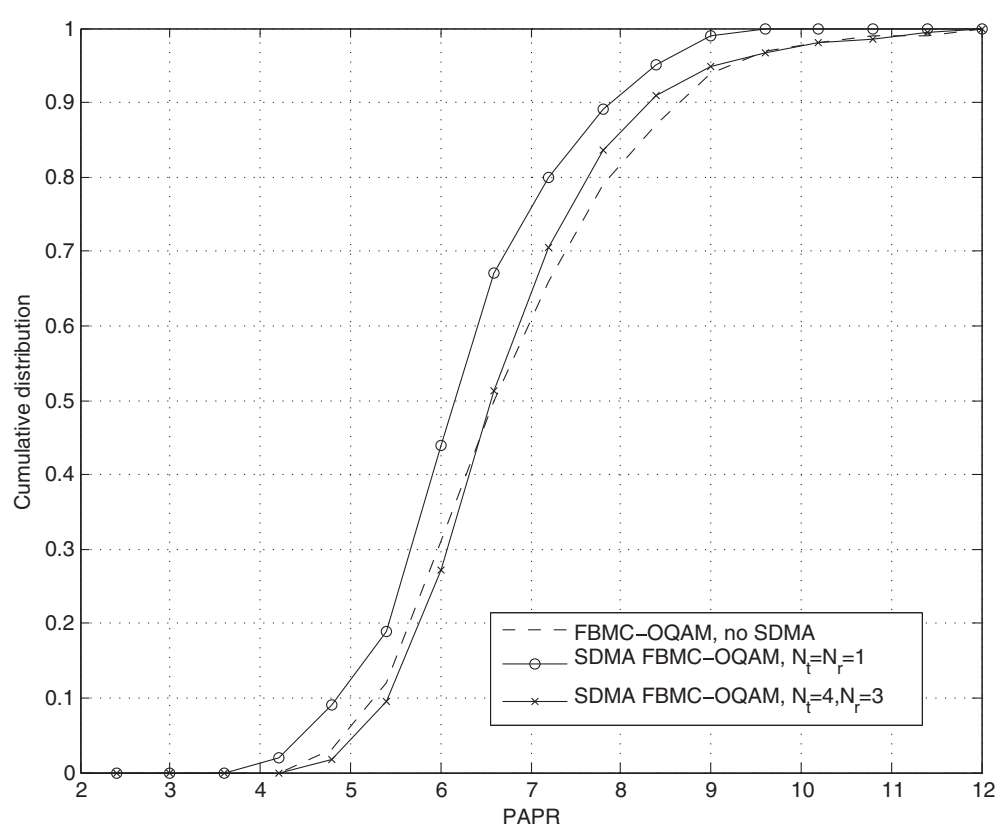

Figure 4 PAPR cumulative distribution. Peak-to-average power ratio (PAPR) cumulative distribution of the FBMC-OQAM system with and without SDMA precoding. Parameters: $M=64$. 


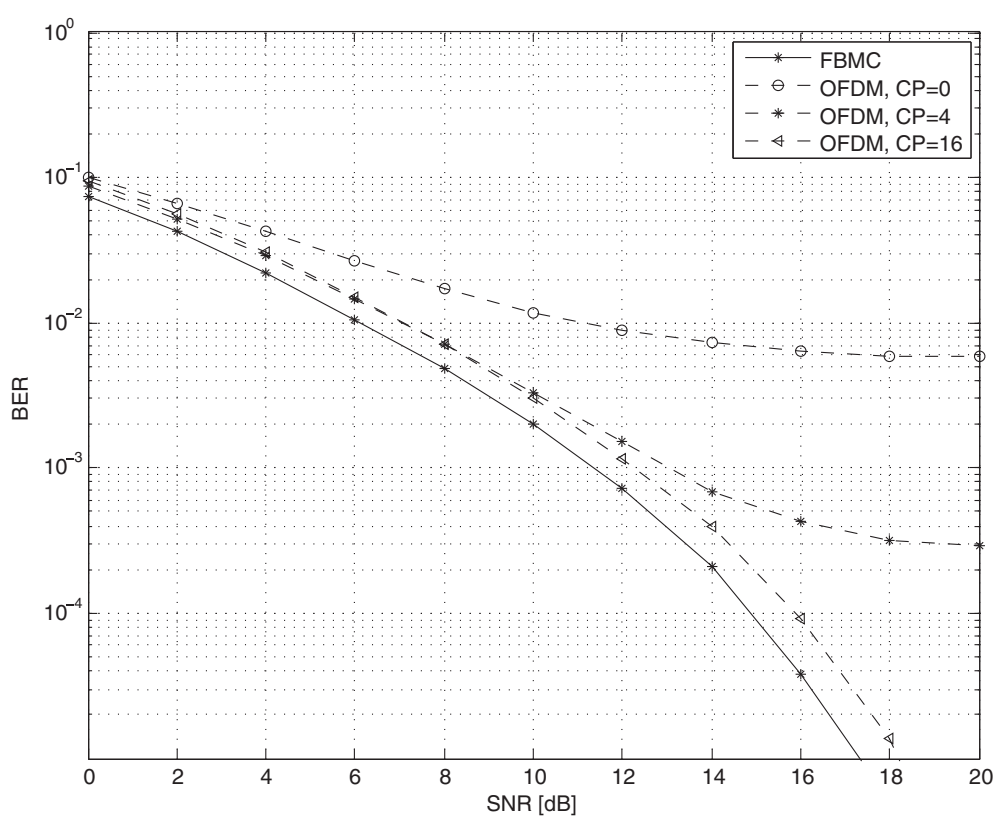

Figure 5 Performance comparison with OFDM system. Bit error rate (BER) of the SDMA FBMC-OQAM and OFDM systems as a function of the signal-to-noise power ratio (SNR). Solid lines: FBMC-OQAM system; dashed lines: OFDM system for a varying cyclic prefix (CP). Parameters: $N_{R}=4$, $N_{T}=3$.

antenna interference. The proposed precoder on the other hand can successfully get rid of all the interference. It delivers therefore a performance approximately independent on the number of subchannels, on the contrary to the single-tap SDMA precoder of performance increasingly limited by the remaining interference when the number of subchannels is reduced.

Figure 7 illustrates the BER for a varying number of receivers. The number of subchannels has been fixed to 64 . The trade-off existing between the diversity and

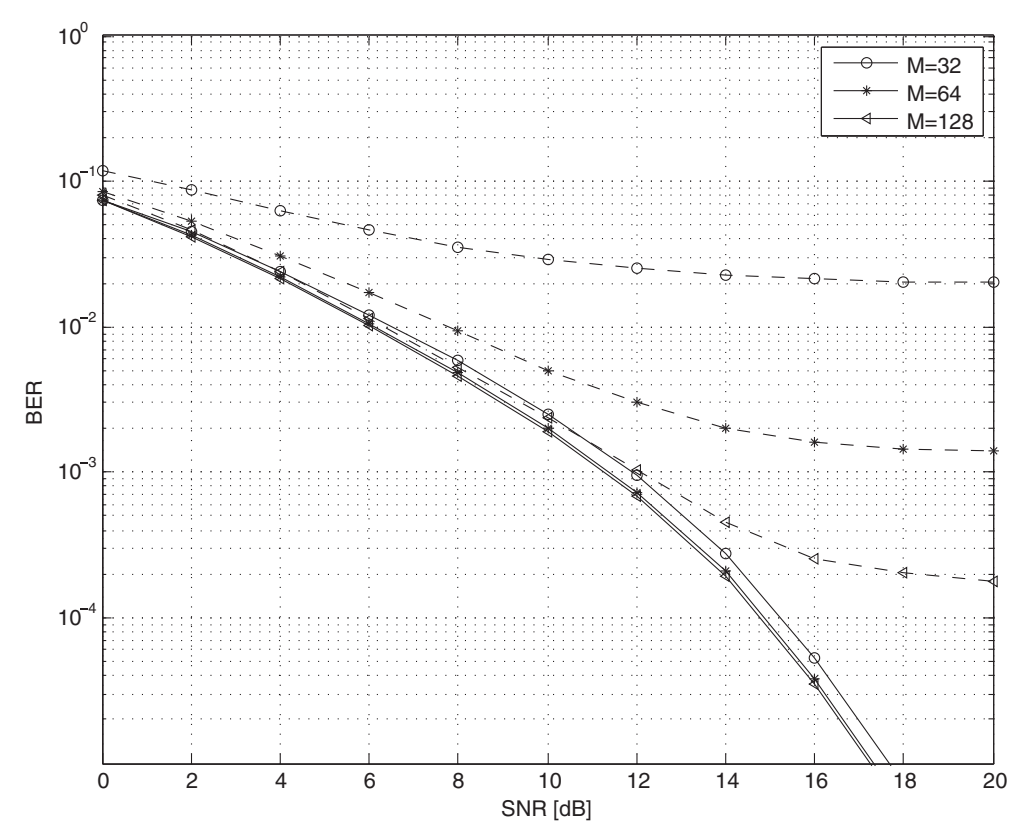

Figure 6 Performance comparison with the state-of-the-art SDMA precoder. Bit error rate (BER) of the SDMA FBMC-OQAM system for a varying number of subchannels M. Solid lines: new SDMA precoder; dashed lines: state-of-the-art single-tap SDMA precoder. Parameters: $N_{R}=4, N_{T}=3$. 


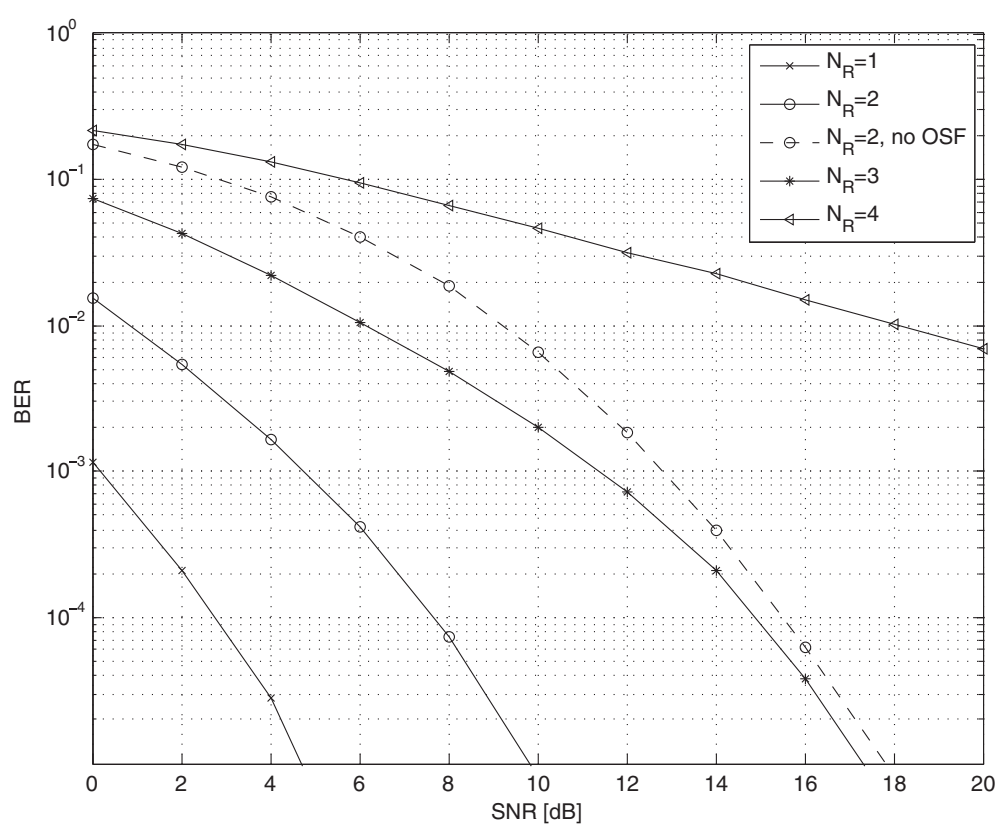

Figure 7 Impact of the number of receivers on the performance. Bit error rate (BER) of the SDMA FBMC-OQAM system for a number of transmitter antennas $N_{T}=4$ and a varying number of receivers $N_{R}$. Solid lines: SDMA precoder designed at fractional rate; dashed line: SDMA precoder designed at symbol rate. Parameters: $M=64$.

spatial multiplexing gains is observed by comparing the solid curves: when the number of receivers decreases in the system, the diversity gain progressively replaces the spatial multiplexing gain (the slope of the curves is steeper but the rate is decreased). Furthermore, the system progressively benefits from an improved power gain (the curves are shifted to the left). The dashed curve illustrates the performance obtained when the SDMA precoder is designed at the symbol rate (instead of at twice the symbol rate) for a number of receivers equal to 2 (so that $N_{T} \geq 2 N_{R}$ ). Compared to the system employing a precoder designed at twice the symbol rate for $N_{R}=2$, the performance is degraded because the system does not benefit anymore from the additional degrees of freedom brought by the precoder over-sampling factor (OSF).

\section{Conclusion}

The objective of the article has been to investigate the combination of the filter-bank multi-carrier (FBMC) modulation, proposed recently for the wireless communications systems because of the improved time/frequency resolution of their waveforms (compared to orthogonal frequency-division multiplexing (OFDM)), with space division-multiple access (SDMA), used in the downlink to separate the users in the spatial domain. The SDMA precoder is built to cancel out the inter-symbol interference on each subchannel, the inter-subchannel interference and the inter-antenna interference. It has the structure of a set of filters used to precode the user streams of information symbols on each subchannel and its adjacent subchannels and on all antennas. Its complexity is therefore much lower than the overall precoder applied on all subchannels simultaneously. The efficiency of the designed scheme has been demonstrated numerically both in terms of multi-access ability and diversity/power gains.

\section{Appendix 1}

\section{Practical computation of the SDMA precoder}

Different methods exist to compute a zero-forcing (ZF) filter for a MIMO channel. In order to compute the $\mathrm{ZF}$ equalizer applied at the receiver on the received samples, the most practical method is probably to build a matrix model of the convolution with the MIMO channel and to compute the corresponding matrix equalizer [20]. For increasing block size, the equalizer matrix approaches the structure of a convolution matrix, so the equalizer filter can be found on the rows of the matrix. The accuracy increases with the size of the matrix model (the parameter $N$ in the model hereafter), but in most cases this method provides a good accuracy for a reasonable complexity [20].

Unfortunately, the method cannot directly be applied to compute the ZF precoder applied at the transmitter on the information symbols as it would require the inversion of an ill-conditioned matrix. The work-around to this problem followed in this article is to compute the ZF 
equalizer of the left-right mirrored MIMO channel and to deduce the ZF precoder of the MIMO channel from it. The left-right mirrored FBMC-OQAM system is obtained by inverting the directions of the arrows in the right part of Figure 2. Neglecting the noise terms, the terms $\mathbf{r}_{k}[n]$ become the inputs and the terms $\mathbf{s}_{k}[n]$ become the outputs of the system. A quick analysis shows that the equations that the filters need to fulfill in order to perform ZF equalization for this left-right mirror systems are identical to the Equations (25)-(29) of the ZF precoder of the initial system, which guarantees that the correct solutions to our initial problem are found using this work-around.

The matrix model is obtained from the continuous time model (23), after inversion of the inputs/outputs, by assuming that finite length- $N$ input sequences are transmitted on finite length- $L$ subchannel impulse responses. In that case, the convolution with the channel impulse responses can be implemented with the matrix multiplication:

$$
\overline{\mathbf{s}}_{k}=\mathbf{G}_{k} \cdot \overline{\mathbf{r}}_{k}
$$

where the input vector of length $10 N_{R} N$ is:

$$
\overline{\mathbf{r}}_{k}=\left[\begin{array}{lllll}
\mathbf{r}_{k-2}^{T} & \mathbf{r}_{k-1}^{T} & \mathbf{r}_{k}^{T} & \mathbf{r}_{k+1}^{T} & \mathbf{r}_{k+2}^{T}
\end{array}\right]^{T}
$$

with:

$$
\mathbf{r}_{j}:=\left[\left(\mathbf{r}_{(1, j)}^{R}\right)^{T} \cdots\left(\mathbf{r}_{\left(N_{R}, j\right)}^{R}\right)^{T} \mid\left(\mathbf{r}_{(1, j)}^{I}\right)^{T} \cdots\left(\mathbf{r}_{\left(N_{R, j}\right)}^{I}\right)^{T}\right]^{T},
$$

for $j=k-2, \ldots, k+2$, and the output vector of length $12 N_{T}(N+L-1)$ is:

$$
\overline{\mathbf{s}}_{k}=\left[\begin{array}{lll}
\mathbf{s}_{k-1}^{T} & \mathbf{s}_{k}^{T} & \mathbf{s}_{k+1}^{T}
\end{array}\right]^{T}
$$

with:

$$
\begin{aligned}
& \mathbf{s}_{i}:=\left[\begin{array}{llll}
\left(\mathbf{s}_{(1, i), 0}^{R}\right)^{T} & \left(\mathbf{s}_{(1, i), 1}^{R}\right)^{T} \quad \cdots \quad\left(\mathbf{s}_{\left(N_{T}, i\right), 1}^{R}\right)^{T}
\end{array}\right. \\
& \left.\begin{array}{llll}
\left(\mathbf{s}_{(1, i), 0}^{I}\right)^{T} & \left(\mathbf{s}_{(1, i), 1}^{I}\right)^{T} & \cdots & \left(\mathbf{s}_{\left(N_{T}, i\right), 1}^{I}\right)^{T}
\end{array}\right]^{T},
\end{aligned}
$$

for $i=k-1, k, k+1$, and the corresponding convolution matrix of size $12 N_{T}(N+L-1) \times 10 N_{R} N$ is: with:

$$
\mathbf{G}_{i, j}:=\left[\begin{array}{c|c}
\mathbf{G}_{i, j}^{R, R} & \mathbf{G}_{i, j}^{R, I} \\
\hline \mathbf{G}_{i, j}^{I, R} & \mathbf{G}_{i, j}^{I, I}
\end{array}\right]
$$

and:

$$
\mathbf{G}_{i, j}^{X, Y}:=\left[\begin{array}{ccc}
\mathbf{G}_{(1, i),(1, j), 0}^{X, Y} & \cdots & \mathbf{G}_{(1, i),\left(N_{R}, j\right), 0}^{X, Y} \\
\mathbf{G}_{(1, i),(1, j), 1}^{X, Y} & \cdots & \mathbf{G}_{(1, i),\left(N_{R}, j\right), 1}^{X, 1} \\
\vdots & \ddots & \vdots \\
\mathbf{G}_{\left(N_{T}, i\right),(1, j), 1}^{X, Y} & \cdots & \mathbf{G}_{\left(N_{T}, i\right),\left(N_{R}, j\right), 1}^{X, Y}
\end{array}\right]
$$

for $X, Y=R, I$. The inner product $\mathbf{s}_{\left(n_{T}, i\right), \rho}^{X}=$ $\mathbf{G}_{\left(n_{T}, i\right),\left(n_{R}, j\right), \rho}^{X, Y} \cdot \mathbf{r}_{\left(n_{R, j}\right)}^{Y}\left(n_{T}=1, \ldots, N_{T} ; n_{R}=1, \ldots, N_{R} ;\right.$ $X, Y=R, I$ and $\rho=0,1)$ implements the convolution of the input sequence $r_{\left(n_{R}, j\right)}^{Y}[l]$ assumed of finite length $N$ :

$$
\mathbf{r}_{\left(n_{R}, j\right)}^{Y}=\left[r_{\left(n_{R, j}\right)}^{Y}[0] \cdots r_{\left(n_{R, j}\right)}^{Y}[N-1]\right]^{T}
$$

with the channel impulse response $g_{\left(n_{T, i}\right),\left(n_{R}, j\right), \rho}^{X}[l]$ assumed of finite length $L$ to produce the output sequences $s_{\left(n_{T}, i\right), \rho}^{X}[l]$ also of finite length $N+L-1$ :

$$
\mathbf{s}_{\left(n_{T}, i\right), \rho}^{X}=\left[s_{\left(n_{T}, i\right), \rho}^{X}[0] \cdots s_{\left(n_{T}, i\right), \rho}^{X}[N+L-1]\right]^{T} .
$$

The $(a, b)$ th entry of the submatrix $\mathbf{G}_{\left(n_{T}, i\right),\left(n_{R}, j\right), \rho}^{X, Y}$ of size $N+L-1 \times N$ is equal to $g_{\left(n_{T}, i\right),\left(n_{R}, j\right), \rho}^{X}[a-b]$ where $g_{\left(n_{T}, i\right),\left(n_{R}, j\right), \rho}^{X, Y}[l]$ are the channel responses assumed different from 0 on the interval $[0, L-1]$ only $(a=0, \ldots, N+$ $L-1 ; b=1, \ldots, N)$. It should be noted that we not only assume that the composite impulse responses (8)-(11) are of finite length to compute the SDMA precoder, but also that they are causal. This is obtained by applying artificially a delay to the impulse responses when the channel matrix (41) is constructed and by removing it from the resulting SDMA precoder.

The zero-forcing equalizer is expressed as $\left(\left(\mathbf{G}_{k}\right)^{H}\right.$. $\left.\mathbf{G}_{k}\right)^{-1} \cdot\left(\mathbf{G}_{k}\right)^{H}$. In the left-right mirrored FBMC-OQAM system, the equalizer provides an estimate $\mathbf{d}_{k}[l]$ of the transmitted vector $\mathbf{r}_{k}[n]$ based on the observation of the received vectors $\mathbf{s}_{k-1}[l], \mathbf{s}_{k}[l]$, and $\mathbf{s}_{k+1}[l]$ as illustrated in the left part of Figure 2 considering that the arrows are oriented in the opposite direction. Similarly to the channel matrix (33), it can be decomposed in $10 N_{R} \times 12 N_{T}$ convolution submatrices of size $N \times N+L-1$. An approximation of the corresponding zero-forcing filters of length $N+L-1$ can be found on the respective rows of the submatrices,

$$
\mathbf{G}_{k}:=\left[\begin{array}{ccccc}
\mathbf{G}_{k-1, k-2} & \mathbf{G}_{k-1, k-1} & \mathbf{G}_{k-1, k} & \mathbf{0}_{4 N_{T}(N+L-1) \times 2 N_{R} N} & \mathbf{0}_{4 N_{T}(N+L-1) \times 2 N_{R} N} \\
\mathbf{0}_{4 N_{T}(N+L-1) \times 2 N_{R} N} & \mathbf{G}_{k, k-1} & \mathbf{G}_{k, k} & \mathbf{G}_{k, k+1} & \mathbf{0}_{4 N_{T}(N+L-1) \times 2 N_{R} N} \\
\mathbf{0}_{4 N_{T}(N+L-1) \times 2 N_{R} N} & \mathbf{0}_{4 N_{T}(N+L-1) \times 2 N_{R} N} & \mathbf{G}_{k+1, k} & \mathbf{G}_{k+1, k+1} & \mathbf{G}_{k+1, k+2}
\end{array}\right]
$$


that improves when the design parameter $N$ increases because the effects on the borders of the finite sequence of symbols become negligible. The SDMA precoder for subchannel $k$ is equal to the equalizer for subchannel $k$ in the left-right mirrored system.

The computation of the zero-forcing filter requires the inversion of the correlation matrix $\left(\mathbf{G}_{k}\right)^{H} \cdot \mathbf{G}_{k}$ which is well-conditioned when the number of rows of $\mathbf{G}_{k}$ is larger than its number of columns $\left(12 N_{T}(N+L-1) \geq 10 N_{R} N\right)$. This is always the case when $N_{T} \geq N_{R}$, independently of the channel length $L$ and the number of symbols $N$. It can also be observed that the zero-forcing filter can be designed at the symbol rate (instead of at twice the symbol rate) when $6 N_{T}(N+L-1) \geq 10 N_{R} N$ which is always the case when $N_{T} \geq 2 N_{R}$.

\section{Endnote}

${ }^{a}$ It is mathematically defined by computing the error matrix on the zero-forcing constraints (25)-(29) due to the inaccuracy of the precoder computation:

$$
\begin{aligned}
\epsilon_{1}= & \mathbf{G}_{k-1, k-2}[l] \otimes \mathbf{P}_{k, k-1}[l] \\
\epsilon_{2}= & \mathbf{G}_{k+1, k+2}[l] \otimes \mathbf{P}_{k, k+1}[l] \\
\epsilon_{3}= & \mathbf{G}_{k-1, k-1}[l] \otimes \mathbf{P}_{k, k-1}[l]+\mathbf{G}_{k, k-1}[l] \otimes \mathbf{P}_{k, k}[l] \\
\epsilon_{4}= & \mathbf{G}_{k+1, k+1}[l] \otimes \mathbf{P}_{k, k+1}[l]+\mathbf{G}_{k, k+1}[l] \otimes \mathbf{P}_{k, k}[l] \\
\epsilon_{5}= & \mathbf{G}_{k-1, k}[l] \otimes \mathbf{P}_{k, k-1}[l]+\mathbf{G}_{k, k}[l] \otimes \mathbf{P}_{k, k}[l] \\
& +\mathbf{G}_{k+1, k}[l] \otimes \mathbf{P}_{k, k+1}[l]-\mathbf{I}_{2 N_{R}} \delta[l] .
\end{aligned}
$$

and by summing the Frobenius norm of all error matrices:

$$
\mathrm{MSE}=\left\|\epsilon_{1}\right\|_{F}+\left\|\epsilon_{2}\right\|_{F}+\left\|\epsilon_{3}\right\|_{F}+\left\|\epsilon_{4}\right\|_{F}+\left\|\epsilon_{5}\right\|_{F} .
$$

\section{Competing interests}

The authors declare that they have no competing interests.

\section{Author details}

${ }^{1}$ OPERA Department, Université libre de Bruxelles, Av. Roosevelt 50, 1050 Brussels, Belgium. ${ }^{2}$ ICTeam, Université catholique de Louvain, Place du Levant 2, 1348 Louvain-la-Neuve, Belgium.

Received: 6 July 2012 Accepted: 1 February 2013

Published: 12 March 2013

\section{References}

1. Y Li, GL Stuber, Orthogonal Frequency Division Multiplexing for Wireless Communications. (Springer, New York, 2006)

2. S Alamouti, A simple transmit diversity technique for wireless communications. IEEE J. Sel. Areas Commun. 16(8), 1451-1458 (1998)

3. A Paulraj, T Kailath, Increasing capacity in wireless broadcast systems using distributed transmission/directional reception (DTDR). U.S. Patent 5345599. Stanford University (1994)

4. BL Floch, M Alard, C Berrou, Coded orthogonal frequency division multiplex. Proc. IEEE. 83(6), 982-996 (1995)

5. C Siclet, P Siohan, in IEEE Globecom, vol. 2. Design of BFDM/OQAM systems based on biorthogonal modulated filter banks (San Fransisco USA, 2000), pp. 701-705

6. DS Waldhauser, JA Nossek, in IEEE International Symposium on Circuits and Systems. MMSE equalization for bandwidth efficient multicarrier systems (Island of Kos Greece, 2006), p. 4

7. A Ikhlef, J Louveaux, in IEEE Pacific Rim Conference on Communications, Computers and Signal Processing. Per subchannel equalization for MIMO FBMC/OQAM systems (Victoria Canada, 2009), pp. 559-564
8. E Kofidis, AA Rontogiannis, in IEEE Personal, Indoor and Mobile Radio Communications. Adaptive BLAST decision-feedback equalizer for MIMO-FBMC/OQAM systems (Istambul Turkey, 2010), pp. 841-846

9. R Zakaria, DL Ruyet, in IEEE Personal, Indoor and Mobile Radio Communications. On maximum likelihood MIMO detection in QAM-FBMC systems (Istambul Turkey, 2010), pp. 183-187

10. R Zakaria, DL Ruyet, in ISWCS. A novel FBMC scheme for spatial multiplexing with maximum likelihood detection (York UK, 2010), pp. $461-465$

11. M Bellanger, in International Symposium on Wireless Pervasive Computing Transmit diversity in multicarrier transmission using OQAM modulation (Santorini Greece, 2008), pp. 727-730

12. C Lele, P Siohan, R Legouable, The Alamouti scheme with CDMA-OFDM/OQAM. EURASIP J. Adv. Signal Process. 2010, 13 (2010)

13. C Lele, DL Ruyet, Decoding schemes for FBMC with single-delay STTC. EURASIP J. Adv. Signal Process. 2010, 11 (2010)

14. M Payaro, A Pascual-Iserte, M Najar, in European Wireless Conference. Performance comparison between FBMC systems under channel uncertainty OFDM (Lucca Italy, 2010), pp. 1023-1030

15. M Najar, M Payaro, E Kofidis, M Tanda, J Louveaux, M Renfors, T Hidalgo, Ruyet D Le, C Lele, R Zacaria, M Bellanger, Deliverable 4.2a - MIMO techniques and Beamforming - Algorithms. Tech. rep. FP7-ICT PHYDYAS PHYsical layer for DYnamic AccesS and cognitive radio (2010)

16. P Vandenameele, L Van der Perre, M Engels, B Gyselinckx, H De Man, A combined OFDM/SDMA approach. IEEE J. Sel. Areas Commun. 18(11), 2312-2321 (2000)

17. S Thoen, L Van der Perre, M Engels, H De Man, Adaptive loading for OFDM/SDMA-based wireless networks. IEEE Trans. Commun. 50(11), 1798-1810 (2002)

18. S Weiss, AP Millar, RW Stewart, in European Signal Processing Conference. Inversion of parahermitian matrices (Aalborg Denmark, 2010), pp. 447-451

19. A Wiesel, YC Eldar, S Shamai, Zero-forcing precoding and generalized inverses. IEEE Trans. Signal Process. 56(56), 4409-4418 (2008)

20. M Miyoshi, Y Kaneda, Inverse filtering of room accoustics. IEEE Trans Acoustics Speech Signal Process. 36(36), 145-152 (1988)

doi:10.1186/1687-6180-2013-46

Cite this article as: Horlin et al.: Interference-free SDMA for FBMC-OQAM.

EURASIP Journal on Advances in Signal Processing 2013 2013:46.

\section{Submit your manuscript to a SpringerOpen ${ }^{\circ}$ journal and benefit from:}

- Convenient online submission

Rigorous peer review

- Immediate publication on acceptance

- Open access: articles freely available online

- High visibility within the field

- Retaining the copyright to your article

Submit your next manuscript at springeropen.com 